\title{
Mobile Life - Innovation in the Wild
}

\author{
Kristina Höök \\ Mobile Life \\ DSV, Stockholm University \\ Forum 100, 16440 Kista, Sweden \\ kia@dsv.su.se \\ http: //www.mobilelifecentre.org
}

\begin{abstract}
After a decade of work in our research labs on mobile and ubiquitous technology, often formed by the early visions of ubiquitous computing, with the urge to move interaction from the desktop out into the wild, these technologies have now moved out into the world - into the wild. We are in the middle of a second IT-revolution, caused by the spread of mobile and ubiquitous services, in combination with a broad consumer-oriented market pull. The first ITrevolution, the introduction and deployment of Internet and the World Wide Web during the 1990's, had a major impact on all parts of our society. As mobile, ubiquitous technology now becomes wide-spread, the design and evaluation of mobile services - i.e. information technology that can be accessed and used in virtually any setting - represents an important business arena for the IT- and telecom industry. Together we have to look for a sustainable web of work, leisure and ubiquitous technology we can call the mobile life.

But what impact does this have on HCI research? In particular, what is our role in innovating new services, new technologies, new interaction models and new ways of living with this technology? Obviously, new methods for design and evaluation of interfaces are needed, especially when those interfaces are not always clearly 'interfaces' anymore, but blend in with various new materials in our environments or even worn on our bodies. Usage situations are shifting, unstable, mobile settings - interaction in the wild. There is a need for design methods that help structure a multitude of different sources of inspiration and fieldwork, and synthesize it into concrete requirements and service or technology concepts. In our work we have used a variety of such methods, such as ethnography as a basis for design, Laban-notation to analyse body behaviours, novel forms of quick sketching of mobile service interaction, cultural probes to understand emotional processes in people's everyday lives, bodystorming for situating ideas in the real world, and the experience clip method for user selfevaluation to evaluate mobile services in their realistic setting. We have also developed our own methods, such as e.g. user-driven innovation - studying extreme or specialised user groups and then innovating services for other user groups based on those experiences.

But we also see trends that will turn these ways of approaching innovation upside down. Producers and consumers blend together in what we name Mobile 2.0 -services, creating content dependent on the mobile setting. Sketching in hardware and software combinations becomes accessible not only to technology experts, but to all. How can HCI-practice change to make the 'digital materials' accessible to all rather than supporting only $\mathrm{HCI}$-experts to develop innovative design?
\end{abstract}


As pointed out in the vision "Being Human: Human-Computer Interaction in the year 2020", HCI needs to orient towards the values shaped by the interaction between technology and people in our everyday lives. As digital, interactive technology enters every aspect of our lives we must do justice to the full complexity of actual human lived experience, where people actively and individually construct meaningful experiences around technology. We might even have to take responsibility for how society is shaped by this second digital revolution - making values such as privacy, autonomy or trust, but also living a good, rich life, explicitly part of our design processes and study methods, creating for a sustainable, human-friendly society.

In the Mobile Life centre, we work around a vision of a ludic society where work mixes with leisure, private with public - a society where enjoyment, experience and play are adopted into all aspects of life. It becomes important to recognise that private and leisure life should not have to be as polished and efficient as your work performance when practices and technology travel between these spheres of our life.

In my talk, I will discuss the implications for academic research in $\mathrm{HCI}$ as well as how this fosters a novel work practice in industry. The ICT and telecom industry will be less focused on identifying needs and more focused on values, in particular, ludic aspects of life. 